\title{
Filosofía de Patricia Benner, aplicación en la formación de enfermería: propuestas de estrategias de aprendizaje
}

\author{
BLANCA ESCOBAR- CASTELLANOS*
}

Universidad de Sonora - México

PATRICIA JARA - CONCHA*

Universidad de Concepción - Chile

Recibido el 20-11-2017; primera evaluación el 13 -12-2018;

segunda evaluación el 21-12-2018; aceptado el 30-01-2019

\section{ReSUMEN}

Los cambios de concepciones de brindar y gestionar el cuidado han planteado la necesidad de modificar los currículos de enfermería para lograr de forma significativa, el perfil de la nueva/o enfermera/o, e incorporarlo al contexto laboral, con conocimientos, habilidades y actitudes, que le permitan desempeñarse eficientemente, con calidad, mayor fundamento científico y conocimientos modernos de gestión. La finalidad del presente estudio es proponer estrategias de aprendizaje desde la perspectiva filosófica de Patricia Benner que permitan su desarrollo y contribuyan a la formación integral del estudiante de enfermería, facilitando su transición de estudiante a profesional. Las estrategias de aprendizaje propuestas promueven e incentivan el desarrollo de habilidades al favorecer la competencia del estudiante a través del análisis, razonamiento y argumentación de fenómenos propios de enfermería, desarrollando una práctica clínica autónoma e innovadora.

Palabras clave: enfermería, educación en enfermería, filosofía en enfermería, estudiantes de enfermería, teoría de enfermería.

\footnotetext{
* Candidata a doctora en Enfermería por Universidad de Concepción, Chile. Con Maestría en Ciencias de Enfermería por Facultad de Enfermería de Nuevo León, Monterrey, México. Maestra de tiempo completo en el Departamento de Enfermería de la Universidad de Sonora en Sonora, México. Jefe del Servicio de Urgencias del Hospital General «Fernando Ocaranza» del ISSSTE en Hermosillo, Sonora, México. Correo: blanca.escobar@unison.mx

** Autora de correspondencia. Doctora y magíster en Enfermería por la Universidad de Concepción, Chile. Directora del programa de Doctorado en Enfermería de la Universidad de Concepción. Profesora titular de la Facultad de Enfermería de la Universidad de Concepción, Concepción, Chile. Correo:pjara@ude.cl
} 


\section{Philosophy of Patricia Benner, application in nursing training: Proposals of learning strategies}

\section{Abstract}

Changes in conceptions of providing and managing care have raised the need to modify nursing curricula to achieve significantly, the profile of the new nurse, and incorporate it into the workplace, with knowledge, skills and attitudes, that allow it to perform efficiently, with quality, greater scientific basis and modern management knowledge. The purpose is to propose learning strategies from the philosophical perspective of Patricia Benner that allow their development and contribute to the comprehensive education of the nursing student facilitating their transition from student to professional. The proposed learning strategies promote and encourage the development of skills by favoring the competence of the student, through the analysis, reasoning and argumentation of own phenomena of nursing, developing an autonomous and innovative clinical practice.

Keywords: nursing, nursing education, philosophy in nursing, nursing students, theory of nursing.

\section{Filosofia de Patricia Benner, aplicaçáo em treinamento de enfermagem: propostas de estratégias de aprendizagem}

\section{ResUMO}

As mudanças nas concepçôes de fornecimento e gerenciamento de cuidados, levantaram a necessidade de modificar os currículos de enfermagem para alcançar significativamente, o perfil da nova/ou enfermeira/o, e incorporá-lo no local de trabalho, com conhecimento, habilidades e atitudes, que permitem que ele desempenhe de forma eficiente, com qualidade, maior base científica e conhecimentos modernos de gestáo. $O$ objetivo é propor estratégias de aprendizagem a partir da perspectiva filosófica de Patricia Benner que permitam seu desenvolvimento e contribuam para a educação abrangente do aluno de enfermagem facilitando a transição do aluno para o profissional. As estratégias de aprendizagem propostas promovem e incentivam o desenvolvimento de habilidades, favorecendo a competência do aluno, através da análise, raciocínio e argumentaçáo de seus próprios fenômenos de enfermagem, desenvolvendo uma prática clínica autônoma e inovadora.

Palavras-chave: enfermagem, educação em enfermagem, filosofia em enfermagem, estudantes de enfermagem, teoria da enfermagem. 


\section{INTRODUCCIÓN}

Las instituciones educativas formadoras de profesionales en enfermería deben afrontar retos que demandan las exigencias contextuales de un mundo globalizado y pluralista del siglo XXI (Vivas, 2010); para así dar respuesta a las necesidades sociales que actualmente prevalecen en el ámbito de la salud. Además de estar acorde a las políticas educativas que prevalecen. Orozco, citado por Irigoyen, Jiménez y Acuña (2011) mencionó que «las políticas educativas en el plano universitario se han orientado a formar profesionales en un nuevo escenario, que implica pensar, trabajar y tomar decisiones en colaboración con otros profesionales, para resolver problemas con un máximo de ejecución eficiente» (p. 244).

En este contexto, Raile (2015) afirmó que desde que enfermería alcanzó el nivel de licenciatura ha ido adquiriendo mayor reconocimiento en la educación superior, concretando el objetivo de un mayor desarrollo integral de conocimiento pertinente como base para la práctica de enfermería. Desde el punto de vista de Gutiérrez-Meléndez (2008), los cambios en las concepciones de brindar y gestionar el cuidado, aunado al ámbito organizacional han planteado la necesidad de modificar los currículos de enfermería para lograr de forma significativa el perfil de la nueva (o) enfermera (o), con la finalidad de que se incorporen al contexto laboral, con conocimientos, habilidades y actitudes que le permitan desempeñarse de forma eficiente, con calidad, mayor fundamento científico y conocimientos modernos de gestión, entre otros, adoptando una postura profesional acorde a la disciplina.

En este sentido, Valenzuela, mencionado por López, Barahona, Estrada, Favela y Cuen (2014), hace referencia a un ejemplo de estos cambios: la transformación del sistema educativo al reestructurar los currículos de enfermería con el modelo educativo de competencias, permite integrar personas cada vez más competentes a nivel laboral, capaces de enfrentar situaciones reales y tomar decisiones de manera correcta. La misma autora menciona que este modelo «conlleva un nuevo enfoque de la evaluación que no solo trate el aspecto cognitivo de las personas, sino que evalúe aspectos prácticos o de transferencia, situaciones integradas y las estrategias metacognitivas» (p. 4).

Es por esto que la formación del estudiante en enfermería de pregrado a nivel mundial se debe fundamentar teóricamente en aspectos relevantes y actualizados del cuidado de la salud humana, integrando el estudio de la experiencia o vivencia de la salud humana (Durán de Villalobos, 2002) en toda actividad de su práctica académica, extendiéndose al ámbito profesional. Es aquí donde surge la importancia de la formación de los futuros enfermeros/as, 
sobre qué tipo de aprendizaje deben desarrollar para realizar una práctica de enfermería integral acorde a las necesidades del paciente/familia/comunidad, sin olvidarse del mundo de las ciencias de la salud y su vertiginoso avance en la era de la modernidad. Una pregunta que puede generarse es ¿cómo podría el estudiante de enfermería adquirir conocimiento durante su formación de pregrado?, que le permita enfrentar y dar solución a las nuevas demandas sociales, políticas, económicas, educativas y en salud a las cuales hará frente en su futuro profesional.

Algunos de estos aspectos o factores han influido en la mayoría de las escuelas de enfermería para que rediseñen los procesos de enseñanza-aprendizaje-evaluación, lo que ha motivado la reestructuración de los currículos en todos los niveles de formación (Vivas, 2010), aunado a la responsabilidad del docente guía, de cómo generar o motivar un aprendizaje significativo que permita desarrollar en el estudiante, la mayoría de las áreas del conocimiento de enfermería.

Sobre la base de lo anterior, este trabajo tiene como objetivo proponer estrategias de aprendizaje desde la perspectiva filosófica de Patricia Benner que permitan el desarrollo y contribuyan a la formación integral del estudiante de enfermería y facilite su transición de estudiante a profesional. Para ello, se realizó una revisión de la literatura en las bases de datos Scientific Electronic Library Online (Scielo), Portal Regional de la Biblioteca Virtual en Salud y Google Académico, además de consultar fuentes escritas (libros y revistas). El periodo de búsqueda comprendió de 2005 a 2016, en los idiomas inglés y español con la finalidad de realizar una lectura interpretativa de la información seleccionada y establecer propuestas específicas.

\section{MarCo teórico}

\subsection{Filosofía de Patricia Benner (teoría fenomenológica)}

Algunos autores abordan la fenomenología como una filosofía y método de investigación para poder captar la experiencia vivida, explorar y captar la experiencia subjetiva del individuo, cómo la está percibiendo y qué significado tiene para este, partiendo de la conciencia (Rubio y Arias, 2013). El enfoque fenomenológico planteado por Patricia Benner permite explicar el significado de los fenómenos de la enfermería a través del análisis, del razonamiento y del argumento o exposición lógica (Brykczynski, 2015). Benner reconoce como fuente de inspiración e influencia los trabajos realizados por Heidegger y Gadamer, quienes incursionaron en el campo de la fenomenología existencial 
y la hermenéutica filosófica, siendo esta una filosofía innovadora y muy influyente que permitió descubrir la naturaleza de la comprensión humana (De la Maza, 2005). Otra influencia importante fue la de Hubert y Stuart Dreyfus quienes la introdujeron en la fenomenología, con el planteamiento del modelo Dreyfus de adquisición y desarrollo de habilidades que utilizó en su obra From Novice to expert (1984) (Benner, 2004).

El modelo de Dreyfus de adquisición y desarrollo de habilidades fue desarrollado a partir del rendimiento situado y aprendizaje experiencial. La atención se centra en el desempeño real y los resultados que se obtienen en situaciones particulares que pueden ser comparadas a través del tiempo. Este modelo no identifica rasgos particulares o talentos de la persona que genera el desarrollo de las habilidades, se centra en el comportamiento demostrado en situaciones donde desarrolla las habilidades y el conocimiento (Benner, Tanner y Chelsea, 2009).

Benner adaptó el modelo de Dreyfus, el cual describe cinco niveles de competencia: principiante, principiante avanzado, competente, eficiente y experto. Este modelo postula que los cambios en los cuatro aspectos de la ejecución tienen lugar en la transición a través de los niveles de adquisición de habilidades: a) se transita de confiar en los principios y normas abstractas a emplear la experiencia específica y pasada; b) se transforma la confianza en el pensamiento analítico basado en normas por la intuición; c) se pasa de percibir que toda la información de una situación es igual de importante a pesar que algunos datos son más importantes que otros; y d) se transcurre de ser un observador individual externo a la situación, a tener una participación activa en la misma. Brykczynski (2015), hace referencia a que, con la aplicación de este modelo a la enfermería, Benner observó que «la adquisición de habilidades basada en la experiencia es más segura y más rápida cuando tiene lugar a partir de una base educativa sólida» (p.120).

En cada una de las etapas de transición, Benner hace referencia a la responsabilidad hacia las personas, la cual se va desarrollando con las habilidades que proporciona la experiencia en la práctica y el conocimiento perceptivo; ambos son claves para desarrollar una práctica intuitiva, la cual permite a los egresados de enfermería buscar evidencias para confirmar los cambios observados en los pacientes y tomar decisiones que permitan guiar sus acciones (Brykczynski, 2015). El conocimiento intuitivo admite la comprensión y la observación clínica especializada, originada por la experiencia (considera el conocimiento y experiencia) ligada en el ser profesional (Blum, 2010).

El estudio de la práctica de enfermería clínica permitió a Benner descubrir y describir el conocimiento que sustentaba la práctica de enfermería, estableció 
la diferencia entre conocimiento práctico y conocimiento teórico. Benner, en Brykczynski (2015), afirmó que el desarrollo del conocimiento en una disciplina práctica «consiste en ampliar el conocimiento práctico (saber práctico) mediante investigaciones científicas basadas en la teoría y mediante la exploración del conocimiento práctico existente desarrollado por medio de la experiencia clínica en la práctica de esta disciplina» (p. 120). En la filosofía de Benner, la experiencia es «el resultado de cuestionar, especificar o negar las nociones preconcebidas en una situación» (p. 121). Esto fue retomado de Heidegger y Gadamer, quienes coincidieron que a medida que el egresado de enfermería gana experiencia, desarrolla una mezcla de conocimiento teórico y práctico lo cual da como resultado el conocimiento clínico, por lo tanto, adquiere pericia ya que es capaz de actuar bajo principios y de modificarlos según una situación real específica. Para Heidegger, el conocimiento práctico fue reconocido como «el que se obtiene cuando un individuo se ve implicado en una situación» (Brykczynski, 2015, p. 121), tomando en cuenta que el individuo comparte conocimientos, habilidades y hábitos procedentes de sus prácticas culturales.

Benner argumenta que la aplicación de una ética relacional basada en la práctica de enfermería permitirá equilibrar el centro dominante sobre derechos y justicia del paciente, ya que la relación entre tomar una decisión clínica y una decisión ética con resultados buenos o malos y lo que se considera una intervención de calidad afecta las acciones y juicios clínicos que el/la estudiante desarrolla durante su proceso de aprendizaje. La importancia de la propuesta realizada por Benner conlleva a poner en práctica una filosofía en enfermería, sobre todo en la formación de estudiantes de enfermería, la cual permite, desde la perspectiva de Leddy y Pepper (citado por León, 2006), «comprender el sistema de creencias y valores de la profesión, incluye una búsqueda constante de conocimientos, por tanto, determina la forma de pensar sobre los fenómenos y en gran medida, de su manera de actuar, lo que constituye un elemento de vital importancia para la práctica profesional» (p. 2).

El modelo de adquisición y desarrollo de habilidades puede ser aplicado en situaciones que conlleven la formación de estudiantes de enfermería y no solo a los ya profesionales de la misma; por ejemplo, los niveles reflejan los cambios por los cuales puede transitar el/la estudiante de enfermería, desde la percepción y compresión de una situación de demanda determinada hasta lograr la experiencia adquirida con la práctica clínica durante su formación o en la integración de su desempeño durante su servicio social o internado (en algunos países) (Benner, 2004).

a) Nivel I principiante o novato. No se tiene experiencia con las situaciones para desempeñar sus tareas, pero se tienen que enfrentar a ellas. 
El/la estudiante de enfermería aprende acerca de las situaciones en términos de atributos objetivos (peso corporal, ingresos, egresos, temperatura corporal, presión sanguínea, pulso entre otros parámetros medibles de la condición del paciente). Este primer nivel podría representar al estudiante en sus primeros semestres de formación (tercero, cuarto semestre o un año previo a su práctica profesional o servicio social).

b) Nivel II principiante avanzado. Es quien puede demostrar rendimiento aceptable. El/la estudiante se ha enfrentado a suficientes situaciones reales o ha sido conducido por un mentor o tutor quien proporciona directrices para el reconocimiento acerca de algunos aspectos clínicos, los componentes de la situación significativa generalmente no son objetivos. El/la estudiante de enfermería debe tener alguna experiencia con situaciones anteriores previas antes que pueda utilizar directrices en situaciones clínicas. El principiante avanzado puede reconocer de forma intuitiva elementos situacionales significativos en la práctica clínica. Este nivel corresponde al estudiante en su última etapa de formación (cuando inicia prácticas profesionales o servicio social). Las siguientes fases $\mathrm{c}$, $\mathrm{d}$ y e requieren de la experiencia para identificar algunos aspectos clínicos significativos, los estudiantes cuentan con conocimientos o competencias indispensables para quien inicia su práctica profesional o servicio social, y que a lo largo de ese periodo logra desarrollar.

c) Nivel III competente. Se tipifica a los egresados de enfermería (pregrado) que han trabajado de dos a tres ańos, que comienzan a ver/observar/analizar sus acciones en términos de metas o planes a largo plazo, contemplan situaciones actuales y futuras más importantes que deben ser consideradas y las que pueden ser ignoradas. Se caracteriza por una sensación de dominio, con capacidad para enfrentar y gestionar diversas contingencias (eventualidades) de enfermería clínica. El egresado de enfermería (a quien se les puede denominar enfermera/o porque están insertos en el ámbito laboral) competente es coherente, previsor, gestiona el tiempo como resultado de la consecución de un cierto nivel de experiencia por medio de la planeación y la previsión, ayuda a alcanzar un nivel de eficiencia y organización. Por lo que el aprendizaje clínico se vuelve crucial para reconocer patrones y determinar que elementos de la situación merecen atención y cuáles no.

d) Nivel IV eficiente. El egresado de enfermería percibe situaciones como totalidades (conjunto), la actuación es guiada por máximas, es capaz de reconocer los principales aspectos y posee un dominio intuitivo de la situación a partir de la información previa que conoce (la experiencia enseńa el dominio), la compresión holística del egresado de enfermería eficiente mejora su toma de decisiones y se muestra más implicado con el paciente y su familia. 
e) Nivel $V$ experto. El egresado de enfermería ya no se basa en el principio analítico (norma, directriz, máxima) para justificar su comprensión de la situación a una acción apropiada. Porque ha adquirido un nivel intuitivo de la situación, es capaz de identificar el origen del problema sin perder tiempo en soluciones y diagnósticos alternativos, muestra dominio clínico y de la práctica basada en los recursos, asimilación del saber práctico, visión general y previsión de lo inesperado. El egresado de enfermería experto posee la habilidad de reconocer patrones de actuación profesional gracias a su amplia experiencia (Benner et al., 2009).

Para lograr que el/la estudiante de pregrado desarrolle habilidades y capacidades que le permitan obtener experiencia, definida esta como "práctica prolongada que proporciona conocimiento o habilidad para hacer algo» (Real Academia Espańola [RAE], 2018) ${ }^{1}$ y pericia, entendida según la RAE (2018) como "sabiduría, práctica, experiencia y habilidad en una ciencia o arte» ${ }^{2}$. Es necesario incorporar metodologías educativas considerando el proceso de aprendizaje de los estudiantes para que logren la adquisición de competencias profesionales, las cuales le permitirán demostrar la asimilación progresiva de conocimientos científicos, actitudes, valores, aptitudes y habilidades para desarrollar la capacidad de abordar los problemas de salud-enfermedad en forma competente (De Souza-Cruz y Mariscal-Crespo, 2016), demostrando un alto sentido de responsabilidad y calidad al otorgar los cuidados a la población.

\subsection{Importancia del modelo de Benner en la educación de enfermería}

La filosofía fenomenológica de Benner con el Modelo de Dreyfus de adquisición y desarrollo de habilidades es aplicable y útil en la educación de los estudiantes de enfermería porque permite el desarrollo de habilidades y conocimientos (Peña, 2010) requeridos para aprender a ser enfermeras/os y desempeñarse como tal, a través del aprendizaje externo que va desarrollándose en los diferentes semestres donde normalmente transita como estudiante. Si se combina con un enfoque interpretativo, permite detallar la práctica de enfermería, porque tiene características universales (no está limitado por edad, enfermedad, salud o ubicación de la práctica de enfermería) (Lyneham, Parkinson y Denholm, 2009).

Los aportes de la investigación generada por Benner et al. (2009) motivaron a los educadores de enfermería a la innovación de la práctica para el/la estudiante

\footnotetext{
Recuperado de http://dle.rae.es/srv/search?m=30\&w=experiencia

Recuperado de http://dle.rae.es/srv/search?m=30\&w=pericia
} 
generando una transformación radical. Además, influyó y permitió realizar un cuestionamiento a la visión que las escuelas de formación de recursos humanos en enfermería poseían acerca de la naturaleza del conocimiento profesional y su relación con la práctica (Medina y Castillo, 2006). La aplicación de la filosofía de Benner ha permitido identificar que las necesidades de aprendizaje en los programas educativos son diferentes en los primeros años del desarrollo del conocimiento clínico que los requeridos en estadios superiores, los estudiantes deben aprender a «ser» enfermeros a través de experiencias de aprendizaje, no solo saber el «qué» y el «cómo», sino el «cuándo».

Dentro de esta identidad, además de permitir la integración del conocimiento científico, se debe integrar la ética diariamente en situaciones únicas y cambiantes, para incentivar el desarrollo de la percepción, el juicio y la acción ética (Medina, 1999). Handwerke (2012) menciona que, para enseńar esta fluidez y conocimientos en la práctica, el profesor/docente debe guiar a los estudiantes a integrar estos aprendizajes durante cada clase y cada experiencia clínica. Las acciones generadas serán el resultado de un proceso de construcción realizado por los estudiantes de forma deliberada y reflexionada sobre los fundamentos teóricos y éticos adquiridos, sus consecuencias inmediatas, la coherencia y legitimidad de los medios para llevarlos a cabo a su práctica educativa. Algunas investigaciones en el ámbito educativo hacen referencia a que el/la estudiante de pregrado debe tener contacto con una práctica clínica similar a la realidad, conducida por profesores/docentes/guías que le permitan desarrollar directrices similares a una situación real.

El uso del modelo en el currículo educativo en estudiantes de enfermería implica el desarrollo de prácticas que puedan favorecer el uso de la toma de decisiones analíticas e intuitivas (forma de conocer y de actuar que no está basado en el razonamiento racional, es saber sin saber cómo se sabe). Para lograr esto es importante que el/la estudiante demuestre la adquisición de conocimientos de forma gradual (de lo simple a lo complejo del cuidado de enfermería), que obtenga suficiente información en su memoria que le permita procesar de forma consciente e inconsciente la manera de ser utilizada en la toma de decisiones (Nyatanga y De Vocht, 2008). Los estudiantes de semestres avanzados, en prácticas profesionales o servicio social, demuestran más experiencia de conocimientos en relación a estudiantes principiantes o de semestres iniciales, los cuales cuentan con información más limitada. Esta forma de construcción de procesamiento se puede fomentar al tomar en cuenta factores como la reflexión, la investigación y la curiosidad clínica que apoye la explicación de las prácticas de expertos en la toma de decisiones clínicas (Lyneham, Parkinson y Denholm, 2008). 
Se rescata la importancia de que a un recién graduado le resulta favorable asignarle una enfermera/o tutor/a competente que lo estimule y explique la práctica en enfermería de modo que la pueda comprender y desarrollar con mayor facilidad. Para mejorar las competencias del recién graduado, es importante incorporar al currículo, la consejería (tutoría) clínica, para favorecer el desarrollo de conocimientos, habilidades y aptitudes del egresado (las cuales en ocasiones son deficientes) para ofrecer un cuidado de calidad de enfermería. La filosofía de Benner permite que el docente/profesor de enfermería brinde al estudiante orientación sobre guías de actuación y comportamientos acerca de la práctica clínica de enfermería en situaciones reales, facilitando su aprendizaje (Ortiz, 2013). El incorporar el modelo de Benner en el sistema educativo de enfermería permite al docente emplear el juicio intuitivo en áreas que afectan al estudiante para la toma de decisiones (Blum, 2010). Muchos docentes en enfermería y medicina han adoptado la rúbrica de evaluación del Modelo de Dreyfus y Dreyfus, como marco conceptual para evaluar la adquisición de habilidades de los alumnos y valorar el progreso a través de las diferentes etapas de principiante, principiante avanzado, competente, eficiente y experto (Carraccio, Benson, Nixon y Derstine, 2008).

\subsection{El estudiante durante su formación en enfermería}

La educación superior, como fenómeno socializador, permite forjar seres humanos idóneos y competentes, para satisfacer las necesidades de una sociedad determinada (Cegueda-Benítez, Gańa-Eretza, Uribe-Ravell y Andrade-Padilla, 2013), como es el caso de formar recursos humanos en enfermería, cuya función sustancial es brindar cuidados para garantizar la salud individual, familiar o comunal contribuyendo a elevar la calidad de vida y reducir los índices de morbi-mortalidad de la población que atienden (Hernández, Lemus, Salgueiro, Morejón y Rodríguez, 2007).

Malvárez (2005) menciona que «la educación juega un papel central en la composición y dinámica de la fuerza de trabajo en enfermería, en la calidad y pertinencia de los cuidados y en el desarrollo de la capacidad institucional en salud» (p. 3). Así mismo, sugiere realizar transformaciones al reformular los sistemas educativos para articular los distintos niveles de formación, reorientar los contenidos curriculares hacia los conocimientos, prácticas y ética de los cuidados de enfermería basados en las necesidades de salud y la condición del sujeto como ser cultural. Además, propone la modernización pedagógica hacia modelos que consideren las condiciones de aprendizaje de adultos, que integren la teoría, la práctica, la investigación y las prioridades de salud, 
promoviendo el pensamiento complejo y el trabajo interdisciplinario, entre otros aspectos.

Soto-Fuentes, Reynaldos-Grandón, Martínez-Santana y Jerez-Yañez (2014) hacen referencia a que la formación del profesional de enfermería es amplia y diversa. En esta, los estudiantes inician la adquisición del conocimiento científico teórico en combinación con el conocimiento práctico que se obtiene de una situación determinada y de las experiencias laborales de los enfermeros asistenciales. La Secretaría de Salud de México (2012) al respecto señala que «asegurar la calidad de la enseñanza a los recursos en formación se vuelve fundamental, al ser la piedra angular para hacer frente a la problemática de salud de la población y contar con profesionales capacitados y especializados» (p. 3). El desarrollo de habilidades se vuelve importante en el acto de brindar el cuidado de enfermería, considerando las necesidades de la persona. Al respecto, Girau y Juanes (2009) mencionan que «las habilidades tienen una estructura integrada por tres elementos: conocimiento específico, sistema operacional específico (acciones) y conocimiento y operaciones lógicas» (p. 1); sin embargo, hace referencia a que las habilidades prácticas conforman el núcleo eje del proceso de enfermería, llevando implícitas un grupo de habilidades elementales que deben cumplirse, tomando en cuenta el estado de dependencia de la persona.

Por ello, es importante identificar qué nivel de habilidad/conocimiento tiene el/la estudiante para establecer o diseñar estrategias específicas de aprendizaje. El aprendizaje, según la RAE, es entendido como «la acción y efecto de aprender algún arte, oficio u otra cosa, permite la adquisición por la práctica de una conducta duradera", Asimismo, etimológicamente, aprender (latín apprehenděre) es adquirir el conocimiento de algo por medio del estudio o de la experiencia. En ese sentido, el/la estudiante de enfermería, a través de su formación académica, va a desarrollar una conducta que le permitirá desenvolverse en un futuro en diversos campos de la enfermería. Cestari y Loureiro (2005) señalaron que la práctica de enfermería se ha convertido en toda la actividad, debido a que desde la formación académica los docentes tienen «la práctica concreta de enfermería como horizonte y objetivo» (p. 2).

Para que el/la estudiante desarrolle un aprendizaje, no solo práctico, sino actitudinal y cognitivo, este debe ser significativo. Al respecto, Acevedo y Beltrán (2014) hacen referencia a que se debe propiciar que «el/la estudiante de enfermería aprenda significativamente lo que implica atribuirle un significado al material objeto de aprendizaje a partir de los conocimientos previos y la actualización de esquemas de conocimiento congruentes con cada situación» (p. 19) que se les presente durante su formación académica. Esto 
permitirá que el/la estudiante de enfermería dé importancia y prioridad real a las necesidades de cada persona.

Para Vivas (2010), el aprendizaje significativo es «el resultado de un proceso de apropiación, interiorización, valoración y reformulación de cualquier objeto de aprendizaje, lo cual conduce a la acción autónoma, innovadora y sustentada (ya sea en la práctica, en la teoría o en ambas)» (p. 2). Mientras que Ausubel, citado por Palomino (1996, p. 2), plantea que «el aprendizaje del estudiante depende de la estructura cognitiva previa que se relaciona con la nueva información", a través de una interacción, por lo que la estructura cognitiva hace referencia al «conjunto de conceptos, ideas que una persona posee en un determinado campo del conocimiento y como la organiza».

Es conocido que el estudiante de enfermería se enfrenta a situaciones clínicas muy diferentes en los entornos clínicos donde realiza su práctica al que se desarrolla en el contexto teórico o áulico (De Souza-Cruz y MariscalCrespo, 2016), estableciéndose como una problemática importante para su desempeño como principiante, debido a que los elementos cognitivos y habilidades que posee pueden ser afectados por las condiciones de su entorno, por lo cual es primordial, entonces, que el docente conozca cuál es la estructura cognitiva del estudiante y su grado de estabilidad, porque cada uno cuenta con una serie de experiencias y conocimientos previos que pueden favorecer o afectar su proceso de aprendizaje. Cuando el estudiante de enfermería llega al sistema educativo de nivel superior, transfiere a sus prácticas clínicas un saber experiencial fruto de su biografía y su ser histórico. Es allí donde el/la estudiante puede emerger e integrarse con los saberes propios de la disciplina al generar y adquirir el nuevo conocimiento (Rivera, 2013).

En el proceso educativo, es importante considerar que el desarrollo del conocimiento en enfermería es una tarea difícil de tratar ya que es una profesión En la que el sujeto de estudio y cuidado es la persona, el cual es un ser humano, dinámico, flexible y en constante transición, por lo que el/la estudiante en formación y el/la enfermero/a profesional deben ser dinámicos al demostrar su desarrollo humanista y múltiples dominios al brindar el cuidado (Gallardo, 2011). Al respecto, Arroyo de Cordero, citado por Paravic (2010), hace referencia que enfermería ha heredado una filosofía de responsabilidad para el cuidado de la vida humana derivada del humanismo, lo que brinda sustento y significado a su práctica profesional con el fin de lograr un nivel satisfactorio de desarrollo y de salud en las personas. Por ello, esta filosofía debe ser transmitida durante toda la formación académica de los estudiantes de enfermería, con el fin de fortalecer el aspecto humano al brindar el cuidado a los pacientes. 
Es relevante considerar durante el proceso formativo «lo que sabe el/la estudiante», de tal manera que pueda establecer relaciones con aquello que debe aprender. El/la estudiante hace conexiones de la nueva información con un concepto relevante preexistente en su estructura cognitiva, generando aprendizaje significativo. Otro elemento importante a considerar es el aprendizaje que se da por observación e imitación de modelos (modelaje) lo cual permite establecer el comportamiento del individuo, siendo un medio poderoso para transmitir valores, actitudes, patrones de pensamiento y comportamiento (Riviére, 1992).

A través del aprendizaje observacional el/la estudiante tiene la posibilidad de desarrollar mecanismos cognitivos complejos y patrones de comportamientos sociales, estimulados por los procesos de la motivación, permitiéndole decidir si lo observado debe ser replicado o no en los campos clínicos donde desarrolla un parte importante de su formación académica. Es bien conocido que en el área de enfermería y de la salud en general el/la egresado/a debe poseer una gran capacidad cognitiva, de resolución de problemas, de interrelacionarse con otras personas, sin dejar de lado su parte espiritual, su formación ética y moral; elementos fundamentales de la competencia «saber ser», lo cual permitirá realizar elecciones y toma de decisiones profesionales (Carrillo, García, Cárdenas, Díaz y Yabrudy, 2013).

Durante la formación de pregrado, el/la estudiante de enfermería, gradualmente, debe ir desarrollando un aprendizaje que le permitirá tomar decisiones en relación a qué tipo de conocimientos debe aplicar en una situación determinada; sobre todo, deberá considerar de quién lo aprendió, cómo lo aprendió, cómo fue trasmitido y qué razones tiene para aplicar el conocimiento, debido a que «el aprendizaje supone un cambio continuo y permanente en el comportamiento, refleja la adquisición de conocimientos/habilidades a través de la experiencia» (Ruiz, 2010, p. 1). Antes de ser un profesional, el/la estudiante de enfermería es una persona inserta en un contexto social e institucional que lo contiene e influye en su identidad. Al mismo tiempo, los estudiantes interactúan en este, con otras personas que a su vez lo pueden transformar debido a la influencia que ejercen en su formación (Soto, 2009).

El aprendizaje significativo, "plantea la interacción humana con su ambiente intentando dar sentido al mundo percibido a través del mundo ya conocido» (Soto, 2009, p. 9). Este aprendizaje en la formación del estudiante de enfermería «permite la interpretación, competencia indispensable que deberá desarrollar un/a enfermero/a como condición previa para brindar cuidados pertinentes y de calidad» (Soto, 2009, p. 10). Paranhos y Mendes citados por Carrillo et al. (2013), mencionan que «el guiar a los estudiantes a su nivel más alto de 
pensamiento independiente y de competencia puede lograrse mejor a través de los métodos de enseñanza que estimulan el pensamiento, hacen hincapié en la resolución de problemas y afectan a la motivación para aprender» (p. 350).

En el campo de la enfermería, existen autores que ofrecen fundamentos teóricos que permiten respaldar el desarrollo de la práctica profesional, como Kerouac, Colliére, Fawcett, Benner, entre otros, favoreciendo la formación de los y las futuras generaciones de enfermeras/o en el desarrollo de habilidades, conocimientos y actitudes para brindar el cuidado. Kerouac, Pepin, Ducharme, Duquette y Major (1996) hacen referencia que para integrar una práctica de enfermería se debe desarrollar un "design de cuidado» porque integra "el qué» del cuidado y "el cómo» de la interacción persona-enfermera/o. El design permite crear un cuidado que requiere de diversos procesos como son la reflexión, la integración de creencias y valores, el análisis crítico, la aplicación de conocimientos, el juicio clínico y la intuición. Su desarrollo le permite crear un cuidado innovador que relaciona la ciencia y el arte de la enfermería, centrado en la persona que transita por diversas experiencias de salud, al estar en continúa interacción con su entorno.

Del mismo modo, Colliére (1997) mencionó que es necesario que en la formación de las y los enfermeras/os se adquieran conocimientos de la vida (aspectos cotidianos y modos de vida), se reduzca la distancia entre las diversas ideologías y los medios que se emplean en los centros educativos y asistenciales, y que el aprendizaje se origine de situaciones experimentadas o vivenciadas para permitir la reflexión en situaciones de diferentes dominios del conocimiento. Además, la formación universitaria introduce al estudiante al proceso interrogativo, obliga al cuestionamiento y lo conduce a la argumentación, le permite adquirir el dominio del pensamiento escrito que son el preludio de las futuras publicaciones.

Benner, en Kérouac et al. (1996), menciona que se debe incentivar el desarrollo de un cuidado individualizado, especifico y contextual tomando en cuenta la experiencia particular de la salud. Para aplicar este cuidado, a las futuras/os enfermeras/os se les deben reconocer diferentes grados de experiencia, desde principiante hasta llegar a ser un experto/a en el cuidado. El grado de experiencia desarrollado exige compromiso, pero también implica poder. Habitualmente, un estudiante o recién egresado de enfermería cuenta con bases teóricas sólidas, pero no cuenta con las capacidades/habilidades suficientes para afrontar una situación clínica real que amerite un actuar expedito y oportuno. Cuando se está en formación, cuenta con el apoyo del docente-tutor, pero en el ámbito profesional debe ser autónomo en la toma de decisiones. Para poder desarrollar «ese poder», debe enfrentarse a situaciones 
de cuidado que le permitan integrar sus capacidades en determinado tipo de pacientes y entorno. Según Benner (citada por Carillo et al., 2013, p. 353) "cuanto mayor tiempo esté en contacto con algún tipo de paciente, mayor es la capacidad de predecir y de actuar basado en la experiencia».

En el cuidado que brinda la enfermería, el poder consiste en reforzar el potencial (to empower) de la persona. Es por esto que se deben potenciar aspectos formativos y actitudinales en el/la estudiante para que pueda cumplir con un perfil de egreso acorde con las necesidades sociales e individuales de los pacientes, realizando valoraciones de enfermería holística (que aborde las esferas biológicas, psicológicas, espirituales y sociales), que le permita identificar ese potencial en las personas para generar su propio cuidado y así fortalecer, mantener, mejorar o iniciar el empoderamiento de ellos mismos. Con respecto a la incorporación del análisis crítico en el currículo de enfermería, Kerouac, citado por Eterovic y Stiepovich (2010), reconoce que se debería preparar a los estudiantes en la toma de conciencia y en la coherencia entre los pensamientos y acciones, tener la disposición a revisarlas y la importancia de compartir estas actividades y reflexiones con otras personas. Kerouac expresa que «la formación contemporánea en cuidados enfermeros consiste en la adquisición de aptitudes intelectuales como el análisis, la resolución de problemas, el juicio y el pensamiento crítico» (p. 11).

En el proceso enseñanza-aprendizaje para abordar el cuidado (lo cual no es una tarea fácil), se requiere de profesores/docentes que seleccionen las estrategias educativas más apropiadas para que el/la estudiante adquiera habilidades y destrezas propias del ejercicio profesional, basados en una concepción de la disciplina (uso de teorías y modelos) que le permita integrar los postulados y valores propios de la enfermería para guiar su propia actuación (MantillaPastrana y Gutiérrez-Agudelo, 2013).

Bahamón (s.f.) afirma que el docente/profesor es el responsable de asegurar que el/la estudiante identifique y emplee las estrategias de aprendizaje, además que desarrolle las habilidades necesarias para llevar a cabo cada actividad de aprendizaje, que le permitan fortalecer su disposición a descubrir sus potencialidades para modificar y generar nuevos conocimientos. Esto implica proponer diversas estrategias educativas innovadoras, tomando en cuenta que los estudiantes aprenden de diversas formas; por lo tanto, se debe estimular el desarrollo de sus competencias para que cumpla con un perfil profesional deseable (Acuña, Silva y Maluenda, 2015). Un ejemplo es mencionado por Martínez-Castillo y Matus-Miranda (2015), quienes hacen referencia que, para incentivar el desarrollo del juicio clínico en el/la estudiante (entendido este como la capacidad de anticiparse a eventos o sucesos y responder de una forma 
pertinente a la situación clínica que se presenta, evitando así secuelas o complicaciones a la persona), es necesario emplear estrategias educativas innovadoras que permitan la integración de conocimientos, habilidades y destrezas en la formación de estudiantes como la simulación clínica de alta eficiencia, la cual brinda experiencia clínica incentivando la adquisición de confianza al ejecutar intervenciones de enfermería especificas con el paciente, disminuyendo el error técnico al momento de realizarlo en una situación real, favoreciendo la reflexión y la toma de decisiones derivados del proceso de valoración e identificación de necesidades y/o problemas presentados en la simulación clínica.

$\mathrm{El} / \mathrm{la}$ estudiante, al adquirir las competencias deseables para el desarrollo de la práctica de enfermería, debe ser capaz de reconocer a la persona en el proceso del cuidado, demostrar interacciones normadas por el respeto mutuo, establecer un compromiso con el tratamiento de enfermería, que sea sensible y profundo, basado en las necesidades reales y potenciales del paciente, establecer relaciones de confianza y mostrar compasión ante la situación de salud que experimenta la persona. Todo ello, a través de una comunicación autentica y actitudes apropiadas para el acto de cuidar (Kérouac et al.). La calidad en la formación del futuro enfermero/a dependerá de los intereses y valores que le admitan desarrollar una actuación profesional de calidad, no solo basada en conocimientos y habilidades (Paravic, 2010).

\section{Estrategias de APRENDizajes PROPUESTAS}

Sobre la base de lo anterior, se plantean algunas estrategias de aprendizajes que pueden promover e incentivar el desarrollo de habilidades, no solo técnicas, y favorecer la competencia en los estudiantes de enfermería.

Las estrategias de aprendizaje propuestas son:

- Favorecer la explicación del significado de los fenómenos de la enfermería a través del análisis, del razonamiento y del argumento o exposición lógica

- Estimular el aprendizaje clínico del estudiante para que aprenda a reconocer patrones y comportamientos de las personas y pueda determinar qué elementos de la situación merecen atención y cuáles no

- En el proceso de aprendizaje, considerar al estudiante como un individuo que comparte conocimientos, habilidades y hábitos procedentes de sus prácticas culturales

- Incentivar a través de su formación académica el desarrollo de un aprendizaje significativo para favorecer una práctica clínica autónoma, innovadora y sustentada 
- Integrar una práctica clínica donde se desarrolle «el qué» del cuidado y «el cómo» de la interacción persona-enfermero/a

- Fomentar la adquisición de aptitudes intelectuales como el análisis, la resolución de problemas, el juicio y el pensamiento crítico

- Guiar a los estudiantes a integrar experiencias de aprendizajes durante cada clase y cada experiencia clínica para incentivar el desarrollo de la percepción, el juicio y la acción ética

- Favorecer la toma de decisiones clínicas analíticas e intuitivas al tomar en cuenta factores como la reflexión, la investigación y la curiosidad clínica

\section{Conclusiones}

La filosofía fenomenológica de Patricia Benner, al aplicar el modelo de adquisición y desarrollo de habilidades, se centra en el comportamiento desarrollado en situaciones reales de la aplicación de habilidades y conocimientos del personal de enfermería; además, permite sustentar el nivel de habilidades que tiene el/la enfermero/a dentro de situaciones específicas de su práctica. Los aportes de Benner han sido estudiados en diversos ámbitos de la disciplina (a nivel asistencial, investigación y educación). Se ha demostrado que puede ser un modelo útil a desarrollar a nivel curricular desde la formación del estudiante de pregrado de la licenciatura en Enfermería, para favorecer el desarrollo del ser y del actuar del mismo como principiante, hasta favorecer el desarrollo de capacidades y habilidades con las cuales debe contar un recién egresado haciéndolo competente profesionalmente, avanzando gradualmente hasta adquirir un cierto nivel de experiencia en la práctica clínica.

La aplicación de este modelo en la educación es vital debido a que tiene características universales para su desarrollo, aunado a estrategias de aprendizaje que motiven al estudiante de enfermería a desarrollar la toma de decisiones sustentada en aspectos éticos y a favorecer la adquisición de pericia en una situación real específica, favorecerá como resultado final un adecuado juicio clínico.

El desarrollo de estrategias de aprendizaje encaminadas a potenciar la reflexión, el análisis crítico, la aplicación de conocimientos técnicos y prácticos humanizados, la intuición y el juicio clínico permitirán crear un «design de cuidado", que sea innovador, centrado en las necesidades reales y potenciales de la persona que transita por una experiencia particular de salud.

El estudiante de enfermería en formación y futuro profesional es un recurso valioso para las instituciones de salud, las cuales demandarán sus servicios profesionales y este deberá demostrar las competencias profesionales 
adquiridas durante su formación universitaria. Gradualmente, irá adquiriendo experiencia personal y profesional ante situaciones reales. Esta experiencia desarrollada exige compromiso, pero al mismo tiempo le brindará el poder de desarrollarse profesionalmente.

\section{REFERENCIAS BIBLIOGRÁFICAS}

Acevedo, M. y Beltrán, N. (2014). Teorías educativas en la enseñanza de enfermería. Revista Cuidarte, 3(6), 16-19.

Bahamón, J. (s.f.). El aprendizaje individual permanente: ¿Cómo lograr el desarrollo de esta capacidad de los estudiantes? Cali: Centro de Recursos para el Aprendizaje. Recuperado de http://www.icesi.edu.co/esn/contenido/pdfs/ cartilla_aprendizaje.pdf

Benner, P. (2004). Using the Dreyfus model of skill acquisition to describe and interpret skill acquisition and clinical judgment in nursing practice and education. The Bulletin of Science, Technology and Society Special Issue: Human expertise in the age of the computer, 24(3), 188-199. https://doi. org/10.1177/0270467604265061

Benner, P., Tanner, C. y Chelsa, C. (2009). Expertise in nursing practice: Caring, clinical judgment and ethics. Segunda ed. Nueva York: Springer. https:// doi.org/10.1891/9780826125453

Blum, C. (2010). Using the Benner intuitive-humanistic decision-making model in action: A case study. Nurse Education in Practice, 10(5), 303-307. https://doi.org/10.1016/j.nepr.2010.01.009

Brykczynski, K. (2015). Cuidado, sabiduría clínica y ética en la práctica de la enfermería. En M. Raile, Modelos y teorias en enfermería (pp. 118-136). Barcelona: Elsevier.

Carraccio, C., Benson, B., Nixon, J. y Derstine, P. (2008). From the educational bench to the clinical bedside: Translating the dreyfus developmental model to the learning of clinical skills. Academic Medicine, 83(8). https:// doi.org/10.1097/ACM.0b013e31817eb632

Carrillo, A., García, L., Cárdenas, C., Díaz, I. y Yabrudy, N. (2013). La filosofía de Patricia Benner y la práctica clínica. Revista Enfermería Global, 32, 346360. https://doi.org/10.6018/eglobal.12.4.151581

Cegueda-Benítez, B., Gaña-Eretza, B., Uribe-Ravell, J. y Andrade-Padilla, M. (2013). Postura de enfermeras asistenciales ante la educación: efecto de una intervención de lectura crítica. Revista Enfermería del Instituto Mexicano del Seguro Social, 21(1), 23-28. 
Cestari, M. y Loureiro, M. (2005). El proceso de enseñanza-aprendizaje en enfermería. Revista Enfermería Global, 7, 1-7.

Colliére, M. (1997). Los cuidados de enfermería... ¿̨a qué precio? En M. Colliére. Promover la vida (pp. 339-353). Madrid: McGraw Hill.

De la Maza, M. (2005). Fundamentos de la filosofía hermenéutica: Heidegger y Gadamer. Revista Teología y Vida, 46, 122-138. https://doi.org/10.4067/ S0049-34492005000100006

De Souza-Cruz, M. y Mariscal-Crespo, M. (2016). Competencias y entorno clínico de aprendizaje en enfermería: autopercepción de estudiantes avanzados de Uruguay. Revista Enfermería Global, 15(41), 121-134. https:// doi.org/10.6018/eglobal.15.1.229551

Durán de Villalobos, M. (2002). Marco epistemológico de la enfermería. Revista Aquichan, 2(1), 7-18.

Eterovic, C. y Stiepovich, J. (2010). Enfermería basada en la evidencia y formación profesional. Revista Ciencia y Enfermería, 16(3), 9-14. https://doi. org/10.4067/S0717-95532010000300002

Gallardo, A. (2011). Evolución del conocimiento en enfermería. Revista Medwave, 11(4).

Girau, D. y Juanes, B. (2009). Las habilidades básicas en los licenciados de enfermería. Odiseo Revista electrónica de pedagogía, 23-52.

Gutiérrez-Meléndez, L. (2008). Formación profesional factor determinante en el ejercicio profesional con calidad en enfermería. Revista Enfermería del Instituto Mexicano del Seguro Social, 16(3), 121-125.

Handwerke, S. (2012). Transforming nursing education: A review of current curricular practices in relation to Benner's latest work. International Journal of Nursing Education Scholarship, 9(1), 1-12. https://doi. org/10.1515/1548-923x.2510

Hernández, J., Lemus, M., Salgueiro, T., Morejón, M. y Rodríguez, N. (2007). Perfeccionamiento de los recursos humanos de enfermería. Revista Ciencias Médicas de Pinar del Río, 11(4), 325-340.

Irigoyen, J., Jiménez, M. y Acuńa, K. (2011). Competencias y educación superior. Revista Mexicana de Investigación Educativa, 16(48), 243-266.

Kérouac, S., Pepin, J., Ducharme, F., Duquette, A. y Major, F. (1996). Propuestas para la práctica enfermera. En S. Kérouac, J. Pepin, F. Ducharme, A. Duquette y F. Major, El pensamiento enfermero (pp. 77-98). Barcelona: Elsevier Masson.

León, C. (2006). Enfermería ciencia y arte del cuidado. Revista Cubana de Enfermeria, 2(4). 
López, J., Barahona, N., Estrada, M., Favela, A. y Cuen, F. (2014). Evaluación de competencias del profesional de enfermería que labora en hospitales públicos y privados. Revista Enfermería Actual en Costa Rica, 27, 1-15.

Lyneham, J., Parkinson, C. y Denholm, C. (2008). Explicating Benner's concept of expert practice: Intuition in emergency nursing. Journal of Advanced Nursing, 64(4), 380-387. https://doi.org/10.1111/j.1365-2648.2008.04799.x

Lyneham, J., Parkinson, C. y Denholm, C. (2009). Expert nursing practice: a mathematical explanation of Benner's 5th stage of practice development. Journal of Advanced Nursing, 65(11), 2477-2484. https://doi. org/10.1111/j.1365-2648.2009.05091.x

Malvárez, S. (2005). Recursos humanos de enfermería: desafíos para la próxima década. Organización Panamericana de la Salud, Organización Mundial de la Salud, 1-4.

Mantilla-Pastrana, I. y Gutiérrez-Agudelo, M. (2013). Procesos de evaluación del aprendizaje del cuidado en la práctica de estudiantes de enfermería. Revista Enfermería Universitaria, 10(2), 43-49. https://doi.org/10.1016/ S1665-7063(13)72628-9

Martínez-Castillo, F. y Matus-Miranda, R. (2015). Desarrollo de habilidades con simulación clínica de alta fidelidad. Perspectiva de los estudiantes de enfermería. Revista Enfermería Universitaria, 12(2), 93-98. https://doi. org/10.1016/j.reu.2015.04.003

Medina, J. y Castillo, S. (2006). La enseñanza de la enfermería como una práctica reflexiva. Revista Texto \& Contexto Enfermagem, 15(2), 303-311. https:// doi.org/10.1590/S0104-07072006000200015

Medina, L. (1999). La formación de enfermeras como práctica reflexiva. En J. Medina, La pedagogía del cuidado, saberes y prácticas en la formación universitaria en enfermería (pp. 191-251). Barcelona: Laertes.

Nyatanga, B. y De Vocht, H. (2008). Intuition in clinical decision-making: a psychological penumb. International Journal of Palliative Nursing, 14(10). https://doi.org/10.12968/ijpn.2008.14.10.31493

Ortiz, D. (2013). Competencias clínicas y nivel de conocimiento de los recién graduados de enfermería al ingresar a la fuerza laboral en hospitales en Puerto Rico (tesis de maestría). Recuperado de http://www.suagm.edu/umet/ biblioteca/UMTesis/Tesis_Salud/2013/D_Ortiz_2013.pdf

Palomino, W. (1996). Teoría del aprendizaje significativo de David Ausubel. II Encuentro de Físicos en la Región Inka;1-9. Recuperado de http://ww2. educarchile.cl/UserFiles/P0001/File/Teor\%C3\%ADa\%20del\%20aprendizaje\%20significativo\%20de\%20David\%20Ausubel.pdf 
Paravic, T. (2010). Enfermería y globalización. Revista Ciencia y Enfermería, 16(1), 9-15. https://doi.org/10.4067/S0717-95532010000100002

Peña, A. (2010). The Dreyfus model of clinical problem-solving skills acquisition: A critical perspective. Medical Education Online, 15, 48-46.

Raile, M. (2015). Introducción a las teorías en enfermería: Historia, importancia y análisis. En M. Raile. Modelos y teorías en enfermería (pp. 1-11). Barcelona, España: Elsevier.

Real Academia Española (2018). Aprendizaje. Diccionario de la Lengua Española. Recuperado de http://dle.rae.es/srv/search?m=30\&w=aprendizaje

Rivera, L. (2013). Saber y experiencia de ellla estudiante de enfermería en sus prácticas de cuidado (tesis doctoral). Recuperado de https://www.tdx.cat/bitstream/handle/10803/132808/01.LNRA_TESIS.pdf;sequence $=1$

Riviére, A. (1992). La teoría social del aprendizaje. Implicaciones educativas. En J. Palacios, A. Marchesi y C. Coll (eds.), Desarrollo psicológico y educación (pp. 1-10). Madrid: Alianza.

Rubio, M. y Arias, M. (2013). Fenomenología y conocimiento disciplinar de enfermería. Revista Cubana de Enfermería, 29(3), 191-198.

Ruiz, Y. (2010). Aprendizaje vicario: implicaciones educativas en el aula. Revista Digital para Profesionales de la Enseñanza, 10, 1-6.

Secretaria de Salud de México (2012). Guía metodológica para la estructuración de planes y programas de la currícula de medicina homeopática a nivel licenciatura en las escuelas y facultades de medicina. Recuperado de http://www. cifrhs.salud.gob.mx/site1/planes-programas/docs/Homeopatia_Guia.pdf

Soto, M. (2009). Enfermeria: modelo de teselaciones para la formación superior. San Luis: Nueva Editorial Universitaria. Recuperado de https://docplayer. es/4888228-Enfermeria-modelo-de-teselaciones-para-la-formacion-superior.html

Soto-Fuentes, P., Reynaldos-Grandón, K., Martínez-Santana, D. y Jerez-Yañez, O. (2014). Competencias para la enfermera/o en el ámbito de gestión y administración: desafíos actuales de la profesión. Revista Aquichan, 14(1), 79-99.

Vivas, N. (2010). Estrategias de aprendizaje. Revista Góndola, 5(1), 27-37. 